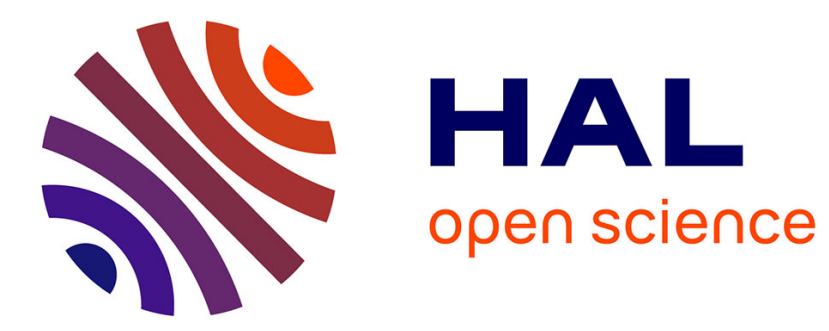

\title{
Resource-Centered Distributed Processing of Large Histopathology Images
}

Daniel Salas, Jens Gustedt, Daniel Racoceanu, Isabelle Perseil

\section{To cite this version:}

Daniel Salas, Jens Gustedt, Daniel Racoceanu, Isabelle Perseil. Resource-Centered Distributed Processing of Large Histopathology Images. 19th IEEE International Conference on Computational Science and Engineering, Aug 2016, Paris, France. hal-01325648

\section{HAL Id: hal-01325648 \\ https://inria.hal.science/hal-01325648}

Submitted on 2 Jun 2016

HAL is a multi-disciplinary open access archive for the deposit and dissemination of scientific research documents, whether they are published or not. The documents may come from teaching and research institutions in France or abroad, or from public or private research centers.
L'archive ouverte pluridisciplinaire HAL, est destinée au dépôt et à la diffusion de documents scientifiques de niveau recherche, publiés ou non, émanant des établissements d'enseignement et de recherche français ou étrangers, des laboratoires publics ou privés.

\section{(1)(1) $\$(0)$}

Distributed under a Creative Commons Attribution - NonCommercial - ShareAlikel 4.0 


\section{Resource-Centered}

Distributed Processing of

Large Histopathology

\section{Images}

Daniel Salas

Daniel Racoceanu
Jens Gustedt

Isabelle Perseil

RESEARCH

REPORT

$\mathrm{N}^{\circ} 8921$

June 2016

Project-Team Camus 



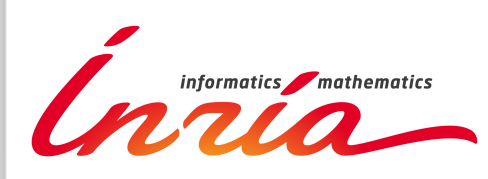

\title{
Resource-Centered Distributed Processing of Large Histopathology Images
}

\author{
Daniel Salas*†‡ \\ Daniel Racoceanu ${ }^{\S}$ \\ Jens Gustedt ${ }^{\dagger \ddagger}$ \\ Isabelle Perseil* \\ Project-Team Camus \\ Research Report n 8921 - June 2016 - 11 pages
}

\begin{abstract}
Automatic cell nuclei detection is a real challenge in medical imagery. The Marked Point Process (MPP) is one of the most promising methods. To handle large histopathology images, the algorithm has to be distributed. A new parallelization paradigm called Ordered Read-Write Locks (ORWL) is presented as a possible solution for solving some of the unwanted side effects of the distribution, namely an imprecision of the results on the internal boundaries of partitioned images. This solution extends a parallel version of MPP that has reached good speedups on GPU cards, but was not scaling to complete images as they appear in practical data.
\end{abstract}

Key-words: parallelization; parallel computing; distributed computing; marked point process; ordered read-write locks; cell nuclei recognition; histopathology

\footnotetext{
* Inserm CISI, Paris, France

$\dagger$ INRIA, Nancy - Grand Est, France

¥ ICube - CNRS, Université de Strasbourg, France

$\S$ Université Pierre et Marie Curie, Paris, France
} 


\section{Calcul distribué centré resources pour de larges images histopathologiques}

Résumé : La détection automatique de noyaux cellulaires est un vrai challenge pour l'imagerie médicale et la lutte contre le cancer. L'un des axes de recherche les plus prometteurs est l'utilisation de Processus Ponctuels Marqués (PPM). L'algorithme tiré de cette méthode a été parallélisé et atteint de bonnes performances d'accélération sur carte GPU. Cependant, cette parallélisation ne permet pas de traiter une lame complète issue d'un prélèvement de biopsie. Il est ainsi nécessaire de distribuer les calculs. Cette distribution entraîne toutefois des pertes de précision au niveau des axes de coupe de l'image. Un nouveau paradigme de parallélisation appelé Ordered Read-Write Locks (ORWL) est une solution possible à ce problème.

Mots-clés : parallélisation; calcul parallèle; calcul distribué; processus ponctuel marqué; ordered read-write locks; détection de noyaux cellulaires; histopathologie 


\section{Introduction}

Breast cancer is the third common cancer all over the world with 1.677 million cases per year [1]. Both its detection and treatment are important concern for public health. E.g, pathologists of the Paris hospital La Pitié Salpétrière have to analyze more than 2000 stained biopsies per day. They evaluate cancer gradation on a scale from 1 to 3 according to 6 criteria. One of the most important criteria is the nuclei size atypia. Along with the cancer progression, nuclei sizes are growing until becoming abnormally big. In 2012, the International Conference on Pattern Recognition organized a contest [2]. Pathologists had to analyze and grade images. A free database of commented slides results from this contest. This is a great opportunity for designing and testing tools that could offer pathologists a second opinion.

Within this context, a team of the Laboratoire d'Imagerie Biomédicale ${ }^{1}$ (LIB) has begun to test techniques to automate cell nuclei detection. An original approach in observing cell nuclei atypia is the Marked Point Process, MPP. In collaboration with the Laboratoire d'Informatique de Paris $6^{2}$ (LIP6), an algorithm has been implemented and then parallelized on CPU with OpenMP and on GPU with CUDA. An acceleration of 22 has been reached with the GPU version. But unfortunately a single GPU is not able support the analysis of a complete slide and so we have to investigate different ways of distributing the algorithm. Section 2 discusses MPP, its existing parallelization and its limits.

In Section 3, we then discuss a conventional distribution method with MPI and a new one using Ordered Read-Write Locks (ORWL) and the benefits of the latter. Section 4 concludes and gives an outlook to future work.

\section{The Marked Point Process for Cell Nuclei Recog- nition in Breast Cancer Images}

\subsection{Marked Point Process Algorithm}

A team from LIP6 has implemented a discretization of the Marked Point Process [3]. The global algorithm is based on a simulated annealing [4] process. The solution tested on each run is the result of a Birth and Death process. The steps of this process are as follows:

Initialization: Image load and resource allocation

Birth Step: A randomly distributed configuration of points is generated. A probability coefficient of birth is decreased in each iteration, to favor convergence.

Mark Step: The best approaching form of a cell nuclei is an ellipse. Points previously created are therefore marked with a wide axis, a short axis

\footnotetext{
${ }^{1}$ See: https://www.lib.upmc.fr/

${ }^{2}$ See: http://www.lip6.fr/
}

$\mathrm{RR} n^{\circ} 8921$ 


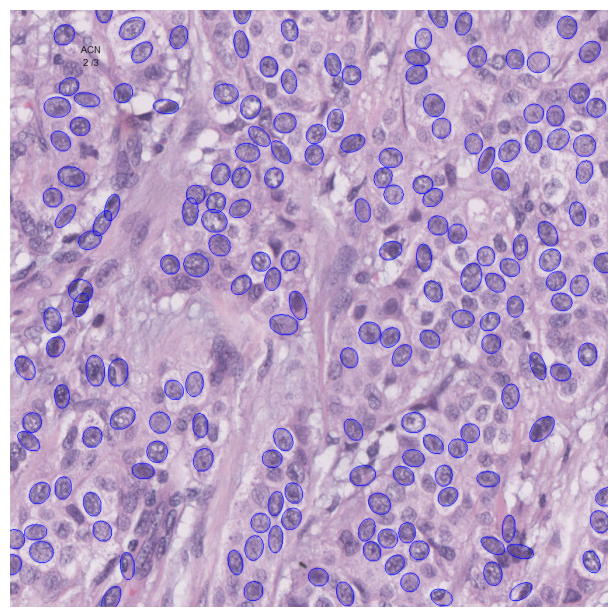

Figure 1: Parallel Birth and Death algorithm result

and an inclination angle. From these parameters, fidelity to the data (also called attachment) is computed, namely the ellipse's accuracy with respect to the underlying image. The Bhattacharyya distance [5] is used to compare the intensity difference between the dark border and the inner cell nuclei.

Neighbors step: A neighbor map is built with the data fidelity values of each pixel. For every pixel of every ellipse, if the map's pixel value is greater than the ellipse's data fidelity value, then the map is updated.

Death Step: A first selection is made between overlapping ellipses. Only the best attached survive. Then a death rate filter is applied on ellipses created from the beginning. This filter is based on the data fidelity value and a death probability. This coefficient is increased in each iteration to favor convergence.

Convergence Test: Convergence is reached when all objects created in the birth phase are killed in the death phase.

\subsection{A Parallel Algorithm for MPP}

LIP6 has also parallelized this algorithm on CPU with OpenMP (see a processed image in Figure 1) and on GPU using CUDA. In each step, the parallelization is straightforward. The loops used to iterate over pixels are distributed to available threads. An initial parallelization problem concerning the death step has been solved using the principle of the neighbors map to test and set the minimal data fidelity values.

Reported parallelization speedups [6] are shown in Table 1. 


\begin{tabular}{|l|c|c|c|}
\hline & Sequential & 4 OpenMP threads & GPU \\
\hline Time & 200 seconds & 60 seconds & 10 seconds \\
Speedup & - & $3.32 \mathrm{x}$ & $22 \mathrm{x}$ \\
\hline
\end{tabular}

Table 1: MPP results on a 1024 by 1024 pixels image

\subsection{Parallel Birth and Death Limitations}

The PBD (Parallel Birth and Death) algorithm is reaching a good speedup on GPU devices. But it is limited to the memory size of the GPU. A complete slide size may be up to 100,000 by 100,000 pixels at full resolution. Modern GPU cards offer $12 \mathrm{GiB}$ memory which is almost the size of our slide. In addition to the image, the application allocates 12 times the number of pixels in arrays of floating points. The total memory needed is about $450 \mathrm{GiB}$. This is far more than the available memory of our GPU.

Therefore, the PBD algorithm has to use subdivisions of the complete slide. On our $12 \mathrm{GiB}$ memory GPU, we could maximally take in charge an image side of about 16,000 px. If we consider analyzing images with a side length of 10,000 pixels, a complete slide would be composed of a hundred images. This leads us to another kind of problem. Subdividing the slide will have the side effect of truncating cell nuclei. The truncated ellipses would not be taken into account for the diagnostic. For an image of x40 magnification, if we consider that our cell nuclei is 80 pixels long, the band of image data that would not be considered correctly would represent a surface of about 3,000,000 px. For the total image this surface represents about $3.2 \%$. If we look at the criteria of nuclei size for cancer gradation [2] (see Table 2),

\begin{tabular}{|c|c|}
\hline grade & $\%$ of atypic nuclei \\
\hline 1 & 0 to $30 \%$ nuclei are bigger \\
2 & 30 to $60 \%$ nuclei are bigger \\
3 & more than $60 \%$ nuclei are bigger \\
\hline
\end{tabular}

Table 2: Breast cancer gradation for atypic nuclei criteria

the unconsidered $3 \%$ of nuclei are sufficient for establishing a diagnostic of grade 1. A cancer may be therefore detected in its early stage. In the following we present a strategy for taking into account carefully the boundary pixels by using a new distributed and parallel computation model.

\section{Distributed PBD Process}

In distributed computing, each processor has its own private memory. This adaptable memory size enables us to manage bigger issues and in particular to analyze bigger images.

$\mathrm{RR} \mathrm{n}^{\circ} 8921$ 


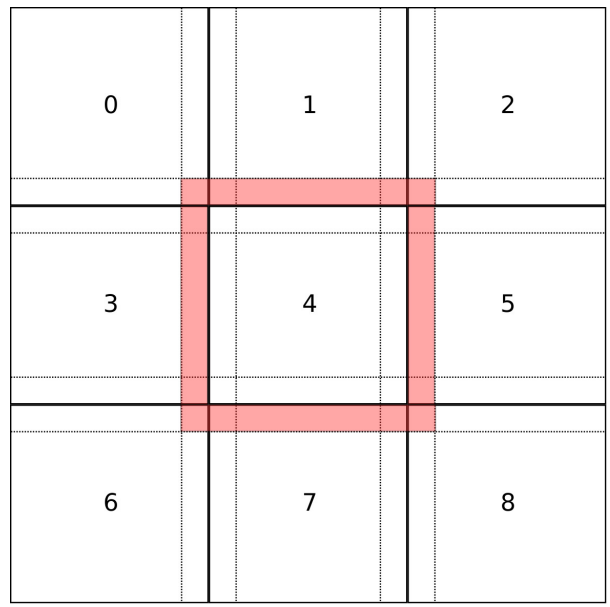

Figure 2: Image distribution strategy

\subsection{Implementation strategy}

The main problem for distributing the algorithm is the neighbor step. For each ellipse data fidelity has to be taken into account to classify for the best attached values. A global map could be distributed among all nodes, according to Figure 2. Every processor could then compute its part of the image locally. At the end of the neighbor step, when the local neighbor map has been drawn, the processors could send each other messages to detect crossing ellipses, according to Figure 3. In the example, Processor 4 sends a message to Processor 1 and informs him of the position and data fidelity value of its ellipses. Processor 1 should be listening asynchronously for this possible message. To avoid deadlock situations, the sends and receives should be non blocking. Then, synchronization barriers must be use to guarantee the coherence of the execution. Such barriers would force the first processor to wait until the last has finished. So, the whole execution would be as slow as the slowest processor. If the work load is not completely balanced, this would add a lot of waiting time, which can be largely sub-optimal. Furthermore, this method could easily encounter a deadlock situation.

Another distribution strategy consists in writing directly into the map of our neighbors. In Figure 2, Processor 4 would access the neighbor map of its surrounding neighbors (the red band). In the example of Figure 3, Processor 4 adds the data fidelity value of its ellipse into Processor 1's neighbor map during its neighbor step. The step in which Processor 1 is at this moment does not matter. The value is taken into account, when Processor 1 proceeds to kill his worst attached ellipses the next time. Then, the map of the whole slide can be updated along with the computing process, no synchronization is necessary. This strategy is possible thanks to a new parallelization paradigm called Ordered 


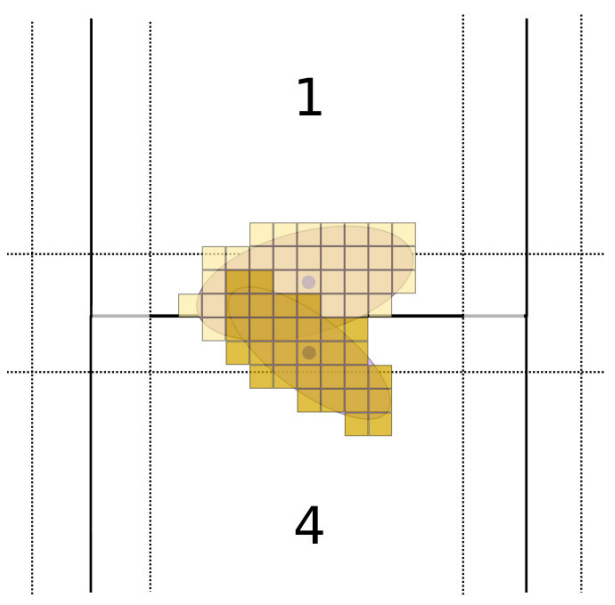

Figure 3: Data fidelity competition

Read-Write Locks ${ }^{3}$ (ORWL).

\subsection{A solution with ORWL}

Before explaining how ORWL will handle the remote write of the neighbors map, we will present its global concepts.

\subsubsection{A new paradigm}

ORWL [7] models parallel and distributed computing by means of tasks. Its particularity is the way in which resources can be shared by the different tasks. Accesses are regulated by a FIFO that guarantees the liveness of the application and the equity of access for the tasks.

\subsubsection{Workflow}

Computation is distributed according to the number of available nodes in a configuration file. The TakTuk library [8] is in charge of sending and collecting files and starting the remote tasks. During a network recognition phase, an address book collects all nodes properties that are necessary to communicate (IP address, port number). Then this book is distributed to all nodes, allowing a point to point communication between all tasks. In a post-computation phase, TakTuk collects the results.

${ }^{3}$ See: http://orwl.gforge.inria.fr/orwl-html/ 
Listing 1: ORWL task launch loop

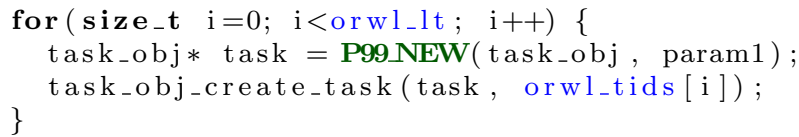

\subsubsection{Tasks}

The number of ORWL tasks is defined at runtime. For each task, a new thread is created and tasks are identified by an unique number orwl_mytid, see Listing 1 for launching tasks.

In this example, the number of tasks is given by orwl_lt. P99_NEW is a macro defined in $\mathrm{P} 9^{4}$. It allocates an element of type task_obj and initializes it with the argument param1. task_obj_create_task executes the block in Listing 2

Listing 2: ORWL handlers definition

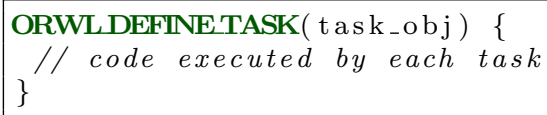

\subsubsection{Handling the data}

ORWL data is held in abstract objects called locations. These are uniquely identifiable entities represented by a global location ID (orwl_myloc). Access to the locations is handled through a queue. To gain control in read or write mode to a location, a handle must insert a request in the queue. Locations have to be declared and an amount of memory has to be allocated as follows:

Listing 3: Parametrization of an ORWL program

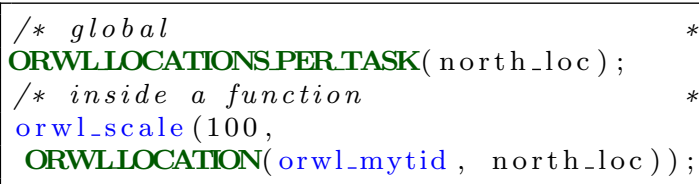

orwl_handle2 for iterative access are initialized and defined as in Listing 4. The macro ORWL_HANDLE2_INITIALIZER initializes the handle object. A memory size is allocated by a call to orwl_scale. Functions orwl_write_insert and orwl_read_insert are used, respectively, to connect an handle with a location in write or read mode. The last parameter of the function provides the initial position of the request in the queue. This strict ordering will schedule data access such that it is deadlock free [9].

\footnotetext{
${ }^{4}$ http://p99.gforge.inria.fr/p99-html/
} 
Listing 4: ORWL handlers definition

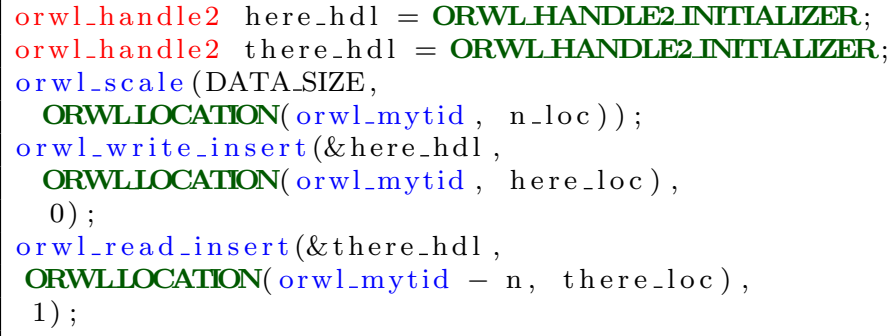

Data is accessible through critical sections as in Listing 5.

Here, the location associated with north_hdl handle is mapped with a pointer to unsigned char. Modifying data through the pointer directly modifies the data of the location itself.

Once a task has gained access to a critical section in write mode, the access is exclusive.

Listing 5: handler data access in write mode

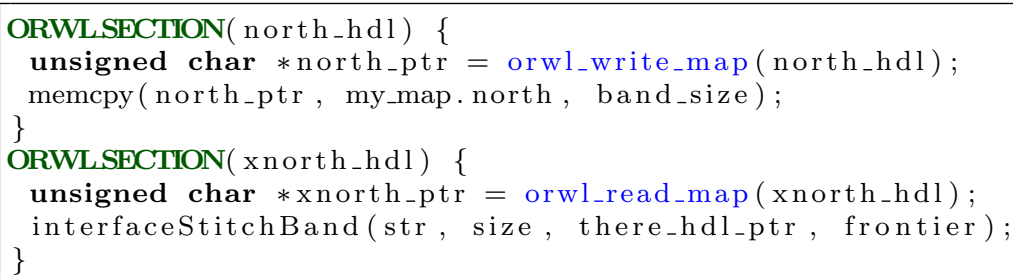

\subsubsection{An ORWL implementation of the MPP}

ORWL is implemented in C. On the other hand, the PBD algorithm has been written in $\mathrm{C}++$. A first step of the implementation consisted in transposing the code from $\mathrm{C}++$ to $\mathrm{C}$. However, we preserved some useful functionalities of the $\mathrm{C}++$ library, for keeping the use of the $\mathrm{CImg}^{5}$ library.

In CImg, an image is represented as a matrix of pixels. The type can be determined during image creation. In our case, we have used images with pixel type unsigned char. Pixels values are accessible through an image.data object. The three color channels $\mathrm{R}, \mathrm{G}$, and $\mathrm{B}$ are stored linearly in a single memory buffer as follows: $R_{0} R_{1} R_{2} \cdots G_{0} G_{1} G_{2} \cdots B_{0} B_{1} B_{2} \cdots$.

Other CImg functions are get_rows and get_columns. They allow to get horizontal and vertical parts of an image. This is used in the distributed PBD algorithm to splice the borders of the neighbor map. The border width is half the wide axis of the ellipses.

\subsubsection{The Distributed PBD algorithm}

Figure 4 shows a workflow of the algorithm.

\footnotetext{
${ }^{5}$ http://cimg.eu/
} 


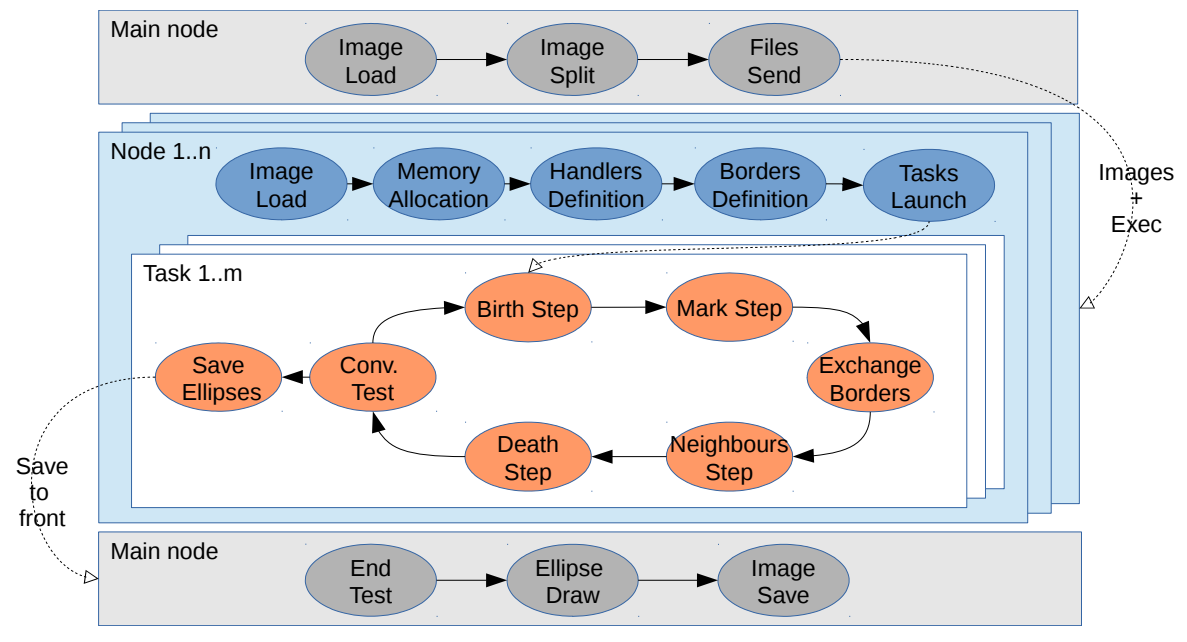

Figure 4: Workflow of PBD ORWL implementation

In an initial phase, a main node loads the entire image and splits it. A Ruby script using TAKTUK sends files to the computing node. Then each node loads its own image. Locations and handlers are defined. Several instances or orwl_tasks can be launched on a single node to parallelize the computation (see Listing 1). After the Mark Step, a new Exchange Border step is added. A first critical section enables a processor to write its border into a location. Then, a second critical section allows to retrieve the borders of the neighbors (Listing 5). Own borders are obtained by slicing the neighbors map. The interfaceStitchBand function adds neighbor borders to the processors neighbor map. Thereby, in the Neighbors Step the computation takes into account neighbors ellipses.

Once a Birth and Death process has converged, ellipse properties are sent to the main node. The final ellipse drawing can be done in a single loop.

\section{Conclusion and future work}

Thanks to the ORWL model, we presented a distributed version of the PBD algorithm that is ready to scale. It allows to properly consider all cell nuclei on the boundary between two partial images. An implementation of this algorithm is in progress and tests will be executed on a cluster of GPU and Xeon Phi accelerators. The final application is designed to map an entire slide in a few minutes. The goal is to let pathologists navigate through the slides, such that they may zoom in and out, just as they used to do with a microscope. Colored ellipses could be shown on a separate layer, helping to detect nuclei size atypia. 


\section{References}

[1] W. H. Organization, "GLOBALCAN 2012: Estimated Cancer Incidence, Mortality and Prevalence Worldwide in 2012," 2012. [Online]. Available: http://globocan.iarc.fr/

[2] I. C. on Pattern Recognition, "MITOS-ATYPIA-14," 2014. [Online]. Available: http://mitos-atypia-14.grand-challenge.org/

[3] C. Avenel, P. Fortin, and D. Béréziat, "Parallel birth and death process for cell nuclei extraction in histopathology images," Jul. 2013, working paper or preprint. [Online]. Available: https://hal.archives-ouvertes.fr/hal-00844001

[4] K. E. Geltman, "The Simulated Annealing Algorithm," 2014. [Online]. Available: http://katrinaeg.com/simulated-annealing.html

[5] F. J. Aherne, N. A. Thacker, and P. I. Rockett, "The bhattacharyya metric as an absolute similarity measure for frequency coded data," Kybernetika, vol. 34, no. 4, pp. 363-368, 1998.

[6] C. Avenel, P. Fortin, and D. Béréziat, "Parallel birth and death process for cell nuclei extraction in histopathology images," 2013.

[7] J. Gustedt and E. Jeanvoine, "Relaxed Synchronization with Ordered Read-Write Locks," in Euro-Par 2011: Parallel Processing Workshops, ser. LNCS, M. Alexander et al., Eds., vol. 7155. Springer, May 2012, pp. 387-397. [Online]. Available: http://hal.inria.fr/hal-00639289

[8] B. Claudel, G. Huard, and O. Richard, "TakTuk, Adaptive Deployment of Remote Executions," in Proceedings of the International Symposium on High Performance Distributed Computing (HPDC). Munich, Germany: ACM, 2009, pp. 91-100. [Online]. Available: https://hal.inria.fr/hal-00788923

[9] P.-N. Clauss and J. Gustedt, "Iterative Computations with Ordered ReadWrite Locks," Journal of Parallel and Distributed Computing, vol. 70, no. 5, pp. 496-504, 2010. [Online]. Available: https://hal.inria.fr/inria-00330024

$\mathrm{RR} \mathrm{n}^{\circ} 8921$ 


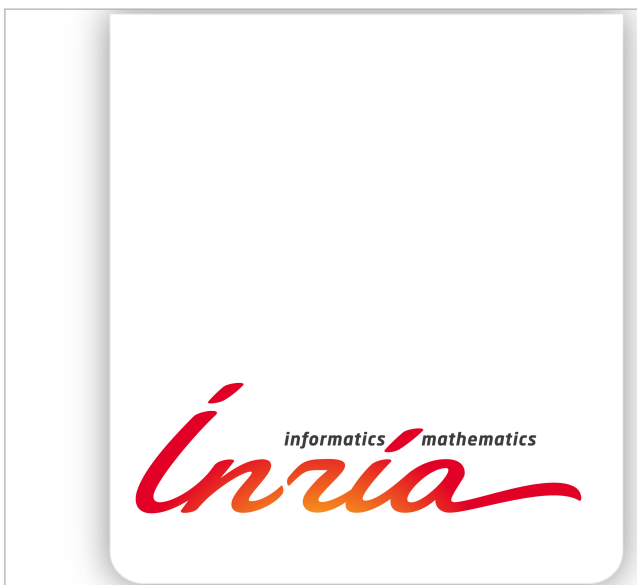

RESEARCH CENTRE

NANCY - GRAND EST

615 rue du Jardin Botanique

CS20101

54603 Villers-lès-Nancy Cedex
Publisher

Inria

Domaine de Voluceau - Rocquencourt

BP 105 - 78153 Le Chesnay Cedex

inria.fr

ISSN 0249-6399 